\title{
Chemokines and Chemokine Receptors in Neurological Disease: Raise, Retain, or Reduce?
}

\author{
Carine Savarin-Vuaillat* and Richard M. Ransohoff* ${ }^{\dagger}$ \\ *Neuroinflammation Research Center, Department of Neurosciences, Lerner Research Institute, and ${ }^{\dagger}$ Mellen Center for MS \\ Treatment and Research, Cleveland Clinic Foundation, 9500 Euclid Avenue, Cleveland, OH 44195.
}

\begin{abstract}
Summary: Chemokines and chemokine receptors comprise a large number of molecules implicated in a wide range of physiological and pathological functions. Numerous studies have demonstrated the roles of chemokines and chemokine receptors: 1) during development, by regulating hematopoiesis, cardiogenesis, and vascular and cerebellar development; 2) during tumor biology, by controlling cell proliferation, angiogenesis, and metastasis; and 3), especially during leukocyte migration, by acting on firm adhesion, locomotion, diapedesis, and chemotaxis. This review focuses on chemokine and chemokine receptor involvement in diverse neurological diseases and their therapeutic potentials. Because of its induction or upregulation during CNS pathologies, members of the chemokine system can be used as biological markers. CXCR4 and CXCL12, by the correlation between their expression and the glioblastoma tumor progression, could be a marker to grade this type of CNS tumor. CCR1, by virtue of
\end{abstract}

specific expression in $\mathrm{A} \beta$ plaques, may be a marker for Alzheimer pathology. Downregulation of CCL2 in cerebrospinal fluid may be a candidate to characterize multiple sclerosis (MS), but needs additional investigation. Moreover, chemokines and chemokine receptors represent interesting therapeutic targets. Using chemokine receptor antagonists, several studies provided exciting findings for potential neurological disease treatment. Chemokine receptor antagonists reduce disease severity in animal models of MS. In glioblastoma, a CXCR4 antagonist (AMD3100) showed an inhibition of tumor growth. Inhibition of chemokine receptor signaling is not the only therapeutic strategy: for example, CXCR4-CXCL12 has anti-inflammatory properties and CX3CL1-CX3CR1 controls neurotoxicity. Thus, chemokine biology suggests several approaches for treating neurological disease. Key Words: Chemokines, chemokine receptors, neurological disease, cell trafficking, marker, antagonist.

\section{INTRODUCTION}

Chemokines-the term is a contraction of chemotactic cytokines-comprise a large family of small $(8-14 \mathrm{kDa})$ basic proteins that display a wide variety of biological and pathological functions. In vitro, the signature assay for chemokines involves stimulation of leukocyte chemotaxis in a concentration-dependent manner. The first chemokine to be described was IL8 (CXCL8), identified in 1987 as a molecule with selective neutrophil chemoattractant properties. ${ }^{1}$ Since then, the chemokine family steadily expanded, now including more than 50 molecules. Chemokines act by binding to G-protein-coupled cell-surface receptors on target cells. The first chemokine receptor (IL8-CXCL8 receptor) was discovered in $1991 .^{2}$ In parallel with their ligands, the interest in chemokine receptors has grown and now nearly 20 chemo-

Address correspondence and reprint requests to: Richard M. Ransohoff, Cleveland Clinic Foundation, Cleveland, $\mathrm{OH}$ 44195. E-mail: ransohr@ccf.org. kine receptors have been described. Chemokine receptors are defined by selective, high-affinity ligand binding coupled with demonstrable biological activity (usually chemotaxis or calcium mobilization).

\section{Chemokines}

The complexity of the chemokine family is due to the large number of component molecules. Moreover, adding confusion to complexity, rapid discovery of new chemokines resulted in various research groups calling the same molecule by different names. This unmanageable situation motivated a consortium, at the Keystone Symposium on Chemokine and Chemokine Receptors in 1999 , to create a systematic nomenclature. ${ }^{3}$

Chemokines are classified into four subfamilies according to the configuration of two positionally conserved cysteine residues near the $\mathrm{NH}_{2}$ terminus. These include the CXC; CC; C; and CX3C subfamilies ${ }^{4,5}$ (FIG. 1) (http://cytokine.medic.kumamoto-u.ac.jp/CFC/CK/ Chemokine.html).

The CXC and CC chemokines are the two major sub- 


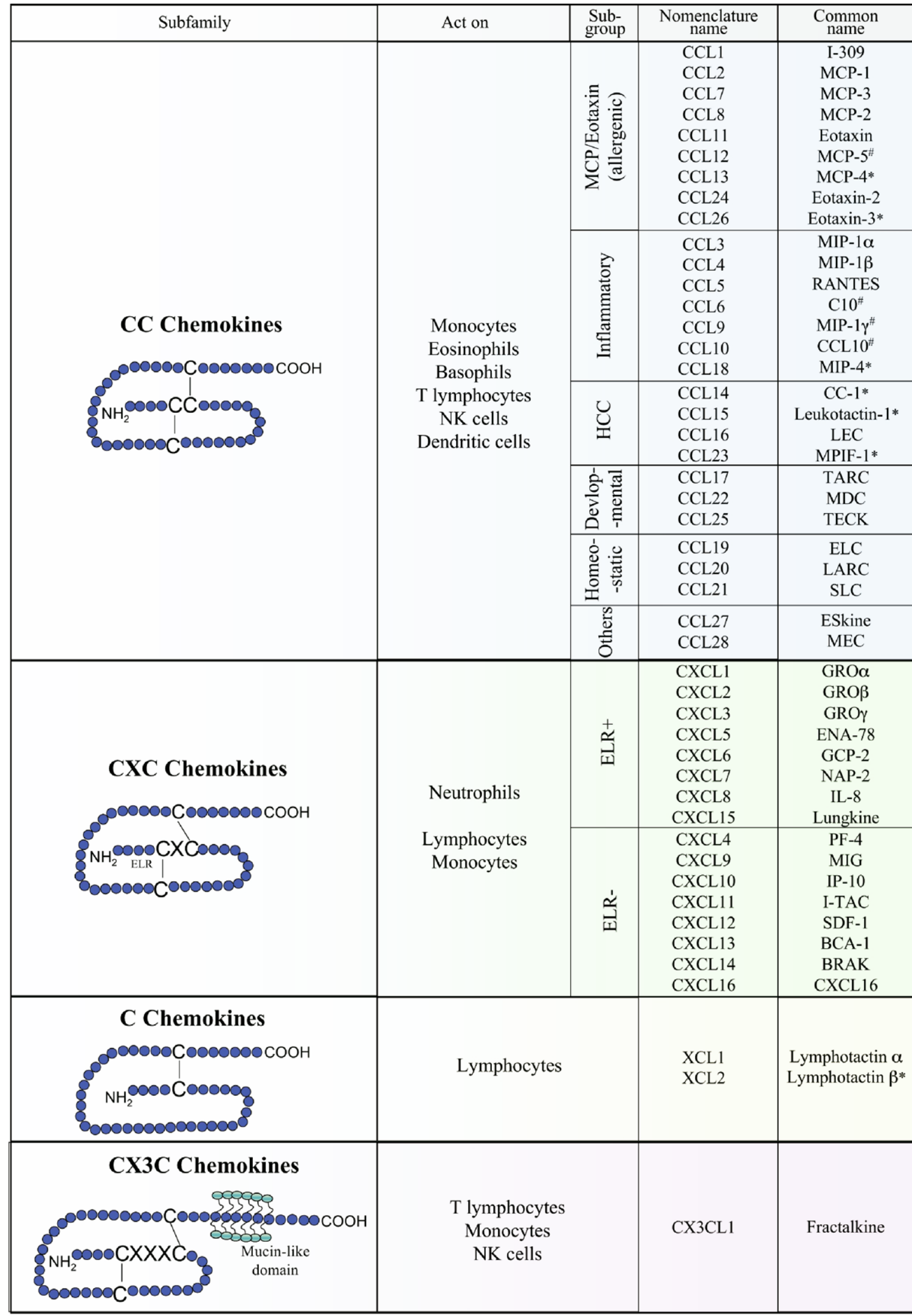

* human only, ${ }^{\#}$ mouse only

FIG. 1. Chemokine nomenclature. 
families. The largest consists of CC chemokines, which are characterized by the adjacent position of the first two cysteine residues. CC subfamily members have a large spectrum of action and can attract monocytes, eosinophils, basophils, $\mathrm{T}$ lymphocytes, natural killer (NK) cells, and dendritic cells. This heterogeneity also extends to their protein sequences and chromosome localization which allow for an informal categorization of this subfamily into various groups, including allergenic (or MCP-eotaxin), inflammatory, HCC (hemofiltrate CC chemokine), developmental, and homeostatic subgroups. ${ }^{5}$ The MCP-eotaxin subgroup includes CCL2 (MCP1), the most extensively studied CC chemokine ${ }^{6}$ (FIG. 1).

The CXC chemokines are characterized by the interposition of a single amino acid $(\mathrm{X})$ between their first two cysteine residues. This CXC subfamily can be subclassified into two other groups, depending on the presence or absence of the sequence motif glutamic acidleucine-arginine (ELR) near the N-terminus (FIG. 1). This structural characteristic of CXC chemokines provides a functional correlation: those containing the ELR motif bind and activate CXCR2, providing specificity for neutrophils and other CXCR2-positive cells, whereas those without the ELR motif have poor chemotactic ability for neutrophils and act primarily on lymphocytes and monocytes.

Unlike these two major subfamilies, the $\mathrm{C}$ and $\mathrm{CX} 3 \mathrm{C}$ chemokines contain two members and one member, respectively. The C chemokines, which comprise XCL1 and XCL2, are distinguished from the other chemokine subfamilies by the presence of only two of the four conserved cysteine residues. ${ }^{7} \mathrm{C}$ chemokines can act on lymphocytes, but not on neutrophils or monocytes.

The sole CX3C chemokine is CX3CL1 (fractalkine). CX3CL1 is characterized by the presence of three amino acids between the first two cysteine residues and also by an extended C-terminal sequence including a mucin-like domain and transmembrane and cytoplasmic regions. According to these structural features, CX3CL1 can be soluble as well as membrane-bound ${ }^{8}$ and acts as an adhesion molecule or a chemoattractant for $\mathrm{T}$ cells, NK cells, and mononuclear phagocytes.

In parallel to this conventional nomenclature, many chemokines can be broadly classified into two functional groups. The first group comprises the homeostatic chemokines, which are expressed constitutively and generally involved in lymphoid organ development and maintenance, as well as immune-surveillance cell trafficking. The second group is the inflammatory chemokines, which are induced by stimuli such as pathogens or inflammatory cytokines and involved in the mobilization of effector cells to sites of inflammation.

\section{Chemokine receptors}

Chemokines exert their biological functions by binding to seven-transmembrane-domain G-protein-coupled receptors on target cells. The chemokine and chemokine receptor nomenclatures are correlated, in that receptors that bind $\mathrm{CC}$ chemokines (for example) are termed CC, followed by ' $R$ ' for receptor and a number that denotes the order of cloning. Thus, the chemokine receptor family comprises the CC (CCR1-10), CXC (CXCR1-7), XCR1 and CX3CR1 receptors (FIG. 2). Chemokine specificity is largely restricted to receptors belonging to the same subgroup. In each subgroup, however, individual chemokines can bind more than one chemokine receptor just as single chemokine receptors can be activated by diverse chemokines. There are isolated instances of monogamous chemokine-chemokine receptor pairs: CXCL13-CXCR5, CXCL16-CXCR6, CCL1CCR8, CCL25-CCR9, and CX3CL1-CX3CR19 ${ }^{9}$ (FIG. 2).

The expression of chemokine receptors is heterogeneous and is not restricted to hematopoietic cells. As with their ligands, chemokine receptor expression can be constitutive or inducible, but also downregulated by exposure to ligand or to activating and differentiating stimuli (FIG. 3). Moreover, some chemokine receptors are widely expressed, whereas others are restricted to certain specific cells or by specific activation or differentiation states. $^{4}$

The activation of chemokine receptors is induced by the recognition and binding of their ligands. Based partly on analogy with other peptide ligands for Gprotein-coupled receptors, the initial recognition between chemokines and their receptors implicates exposed loops between the $\beta$-strands of the chemokine fold and the chemokine receptor extracellular protruding regions. Next, the $\mathrm{N}$ terminal region of the chemokine initiates the activation of the receptor, ${ }^{10}$ which is followed by the internalization of the complex. G proteins are then activated, driving dissociation of their heterotrimers into $\alpha$ and $\beta \gamma$ subunits. Next, various signaling cascade effectors are activated, including phospholipase C (PLC), MAP kinases, or phosphatidyl inositol-3OH kinase (PI-3K), ${ }^{11,12}$ which leads to functional outcomes induced by chemokine receptor signaling (FIG. 3).

Chemokine receptor activation and signaling are strictly controlled by desensitization, which prevents overstimulation of cells and inappropriate response ${ }^{12,13}$ (FIG. 3). Chemokine receptor desensitization implies a multistep process and a complex of proteins, including G-protein-coupled receptor kinases (GRKs) and $\beta$-arrestins. This process starts with the phosphorylation of the chemokine receptor C-terminal tail by GRKs, which increases the receptor affinity for $\beta$-arrestin proteins. The binding of $\beta$-arrestins to chemokine receptors prevents any other interaction between the receptor and G proteins. Then, the GRK- $\beta$-arrestin complex promotes the 


\begin{tabular}{|c|c|c|c|c|c|c|c|}
\hline & \multirow{3}{*}{$\begin{array}{l}\text { Receptor } \\
\text { CCR1 }\end{array}$} & \multicolumn{2}{|c|}{ Express on } & \multicolumn{4}{|c|}{ Ligands } \\
\hline \multirow{11}{*}{ 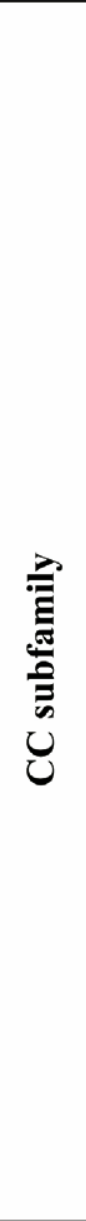 } & & \multirow{2}{*}{$\begin{array}{c}\text { Granulocyte cells } \\
\text { Neutrophils } \\
\text { Eosinophils } \\
\text { Basophils }\end{array}$} & \multirow{2}{*}{$\begin{array}{c}\text { Mononuclear cells } \\
\text { Macrophage } \\
\text { Immature DC } \\
\text { T lymphocytes } \\
\text { NK cells }\end{array}$} & \multicolumn{4}{|c|}{ Affinity } \\
\hline & & & & $\begin{array}{l}\text { CCL3 } \\
\text { CCL5 } \\
\text { CCL7 } \\
\text { CCL13 }\end{array}$ & $\begin{array}{l}\text { CCL14 } \\
\text { CCL15 } \\
\text { CCL23 }\end{array}$ & CCL8 & $\begin{array}{l}\text { CCL4 } \\
\text { CCL2 }\end{array}$ \\
\hline & CCR2 & Neutrophils & $\begin{array}{c}\text { Macrophage } \\
\text { T lymphocytes } \\
\text { B lymphocytes } \\
\text { NK cells }\end{array}$ & $\begin{array}{l}\text { CCL2 } \\
\text { CCL7 } \\
\text { CCL8 }\end{array}$ & $\begin{array}{l}\text { CCL12 } \\
\text { CCL13 }\end{array}$ & & \\
\hline & CCR3 & $\begin{array}{l}\text { Eosinophils } \\
\text { Basophils }\end{array}$ & T lymphocytes & $\begin{array}{l}\text { CCL11 } \\
\text { CCL13 }\end{array}$ & $\begin{array}{l}\text { CCL15 } \\
\text { CCL24 }\end{array}$ & $\begin{array}{l}\text { CCL5 } \\
\text { CCL7 } \\
\text { CCL8 } \\
\text { CCL26 }\end{array}$ & \\
\hline & CCR4 & & $\begin{array}{l}\text { Immature DC } \\
\text { T lymphocytes } \\
\text { NK cells }\end{array}$ & $\begin{array}{l}\text { CCL17 } \\
\text { CCL22 }\end{array}$ & & & \\
\hline & CCR5 & & $\begin{array}{l}\text { Macrophages } \\
\text { DC } \\
\text { T lymphocytes } \\
\text { B lymphocytes }\end{array}$ & $\begin{array}{l}\text { CCL3 } \\
\text { CCL4 }\end{array}$ & $\begin{array}{l}\text { CCL5 } \\
\text { CCL8 }\end{array}$ & & $\begin{array}{l}\text { CCL7 } \\
\text { CC11 } \\
\text { CC13 }\end{array}$ \\
\hline & CCR6 & & $\begin{array}{l}\text { Immature DC } \\
\text { T lymphocytes } \\
\text { B lymphocytes }\end{array}$ & \multicolumn{2}{|c|}{ CCL20 } & & \\
\hline & CCR7 & & $\begin{array}{c}\text { Mature DC } \\
\text { T lymphocytes } \\
\text { B lymphocytes }\end{array}$ & \multicolumn{2}{|c|}{$\begin{array}{l}\text { CCL19 } \\
\text { CCL21 }\end{array}$} & & \\
\hline & CCR8 & & $\begin{array}{l}\text { Macrophages } \\
\text { T lymphocytes } \\
\text { B lymphocytes }\end{array}$ & \multicolumn{2}{|c|}{ CCL1 } & & \\
\hline & CCR9 & & T lymphocytes & \multicolumn{2}{|c|}{ CCL25 } & & \\
\hline & CCR10 & & T / B lymphocytes & CCL27 & CCL28 & & \\
\hline \multirow{7}{*}{ 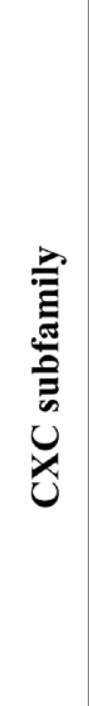 } & CXCR1 & Neutrophils & Macrophages & \multicolumn{2}{|c|}{ CXCL8 } & CXCL6 & CXCL5 \\
\hline & CXCR2 & $\begin{array}{l}\text { Neutrophils } \\
\text { Eosinophils }\end{array}$ & Macrophages & $\begin{array}{l}\text { CXCL1 } \\
\text { CXCL2 } \\
\text { CXCL3 }\end{array}$ & $\begin{array}{l}\text { CXCL5 } \\
\text { CXCL7 } \\
\text { CXCL8 }\end{array}$ & CXCL15 & \\
\hline & CXCR3 & & $\begin{array}{l}\text { T lymphocytes } \\
\text { B lymphocytes }\end{array}$ & \multicolumn{2}{|c|}{$\begin{array}{l}\text { CXCL9 } \\
\text { CXCL10 } \\
\text { CXCL11 }\end{array}$} & & \\
\hline & CXCR4 & Neutrophils & $\begin{array}{l}\text { Macrophages } \\
\text { DC } \\
\text { T lymphocytes } \\
\text { B lymphocytes }\end{array}$ & \multicolumn{2}{|c|}{ CXCL12 } & & \\
\hline & CXCR5 & & $\begin{array}{l}\text { T lymphocytes } \\
\text { B lymphocytes }\end{array}$ & \multicolumn{2}{|c|}{ CXCL13 } & & \\
\hline & CXCR6 & & T lymphocytes & \multicolumn{2}{|c|}{ CXCL16 } & & \\
\hline & CXCR7 & & & CXCL11 & CXCL12 & & \\
\hline 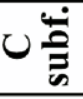 & XCR1 & & $\begin{array}{l}\text { T lymphocytes } \\
\text { NK cells }\end{array}$ & & & & \\
\hline$\bigcup_{0} \frac{1}{0}$ & CX3CR1 & & $\begin{array}{l}\text { Macrophages } \\
\text { T lymphocytes } \\
\text { NK cells }\end{array}$ & $\mathrm{CX}$ & & & \\
\hline
\end{tabular}

FIG. 2. Chemokine receptor nomenclature. 


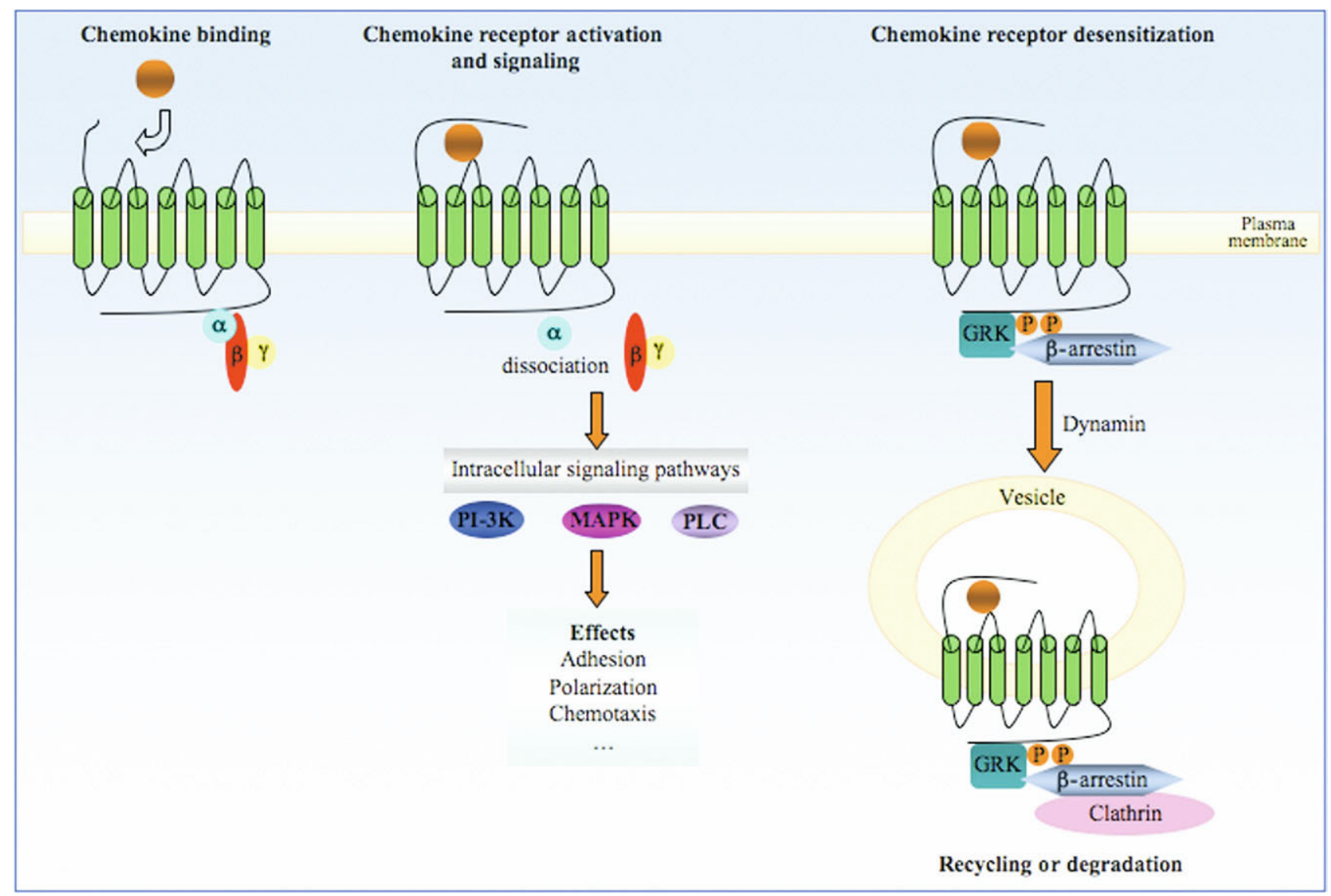

FIG. 3. Chemokine receptor signaling. Following ligand recognition and binding, chemokine receptor signaling starts with $\mathrm{G}$ protein activation, characterized by the dissociation of their heterotrimers into $\alpha$ and $\beta \gamma$ subunits. Downstream effectors include MAPK, PI-3K, and PLC. This signaling cascade leads to varied functional outcomes, such as adhesion, polarization, chemotaxis, and the like. Desensitization starts with the $\mathrm{C}$-terminal chemokine receptor tail phosphorylation, which increases the affinity of $\beta$-arrestin proteins for the receptor and prevents further interaction between chemokine receptors and $\mathrm{G}$ proteins. Clathrin-mediated internalization of the ligated chemokine receptor into vesicles is promoted by the GRKs- $\beta$-arrestin complex and requires the GTPase activity of dynamin. The internalized chemokine receptor is then degraded or recycled. Abbreviations: GRKs, G protein-coupled receptors kinases; MAPK, mitogen activated protein kinase; PI-3K, phosphatidyl inositol-3OH kinase; PLC, phospholipase C.

internalization of the chemokine receptor into vesicular compartments for degradation or recycling. In addition, several studies have suggested that GRKs and $\beta$-arrestins could also modulate chemokine receptor signaling by acting as adaptors for effectors such as PI-3K or MAP kinases. ${ }^{13}$

\section{PLEIOTROPIC FUNCTIONS OF CHEMOKINES AND CHEMOKINE RECEPTORS}

Initially studied because of their roles during inflammation, chemokines and chemokine receptors are now often studied in the broader contexts of leukocyte trafficking from circulation to tissues during development, immune surveillance, and inflammation. This leukocyte migration across the endothelium and the basement membrane is highly controlled and includes multiple steps: tethering, rolling, activation, firm adhesion, and diapedesis. ${ }^{14,15}$ Molecules such as selectins, integrins, and chemokines are involved in the dialogue between leukocytes and endothelial cells.

Specifically, chemokines affect the firm adhesion of leukocytes under flow conditions by integrin activation, which leads to conformational changes of the integrins that increases their affinity for their endothelial receptors and paves the way for leukocyte extravasation. ${ }^{16}$ Chemokines also regulate leukocyte-endothelial interactions at the levels of locomotion and diapedesis. Indeed, apical chemokines can promote locomotion of leukocytes to interendothelial junctions. Then, under fluid shear forces, morphological deformations of these leukocytes occur, resulting in the extension of chemokine receptor-enriched processes through junctions. ${ }^{17}$ These morphological changes facilitate leukocyte exposure to abluminal chemokines and mediate diapedesis along a chemoattractant gradient.

In addition to their implication in leukocyte firm arrest, locomotion, and diapedesis, chemokines direct 
cell migration in a concentration-dependent manner. Under physiological conditions, leukocyte chemotaxis is implicated in the permanent cell trafficking among bone marrow, blood, tissues, and lymphoid organs. Mature dendritic cell (DC), T cell, and B cell homing and recirculation are regulated by CCL19, CCL21, and CXCL13 expressed variously in lymphatic vessels, high endothelial venules (HEVs), and secondary lymphoid organs. ${ }^{3,9,18,19}$ Thus, after CCR7 acquisition during maturation, DC are able to migrate into the T-cell zones of draining lymph nodes in response to CCL19 and CCL21 produced by lymphatic vessels. In the same way, naïve $\mathrm{T}$ cells, characterized by the expression of CCR7, move to lymph nodes in response to CCL19 and CCL21 through HEVs. ${ }^{20}$ In parallel, the migration of $\mathrm{B}$ cells, which express CXCR5, to lymphoid organs is driven by CXCL13, produced by follicular stromal cells. ${ }^{21}$

Conjointly with these homeostatic functions, chemokines are implicated in leukocyte chemotaxis during a wide range of diseases, especially those with inflammatory components. Thus, chemokines are responsible for the accumulation and activation of leukocytes in tissues. The infiltrated cell type depends on the specificity of chemokine production and chemokine receptors present on nearby cells. For example, rheumatoid arthritis is characterized by monocyte and $\mathrm{T}$ cell infiltration into synovial tissues in response to CCL2, CCL3, and CCL5. ${ }^{9}$ During obesity-induced diabetes, the involvement of CCL2 in the impairment of insulin-dependent glucose uptake in adipocytes via macrophage recruitment, as well as the implication of CCL3, has been demonstrated. ${ }^{22,23}$ CXCL9, CXCL10, and CXCL11 have been implicated in type $1 \mathrm{~T}$ helper cell recruitment to inflamed skin during psoriasis and dermatitis. ${ }^{24}$

These are only three examples of diseases with chemokine-mediated cell recruitment and inflammation, but chemokines and chemokine receptors are involved in a large variety of pathologies, such as atherosclerosis, asthma, Crohn's disease, bacterial pneumonia, acute respiratory distress syndrome, bacterial or viral meningitis, sarcoidosis, and tuberculoid leprosy, as well as a wide variety of neurological diseases (addressed later in this review).

Other studies have also identified roles for chemokines during development, especially the critical role of CXCR4. Based on gene-targeted mice, varied studies have implicated CXCR4 in the survival of the embryo, earliest stage of B lymphopoiesis, hematopoiesis, vascular development, cardiogenesis, and cerebellar development. ${ }^{25,26}$

In addition, chemokines are involved in tumor biology by acting on cell proliferation, tumorigenesis, angiogenesis and metastasis. Several chemokines regulate cell proliferation. CXCL1, CXCL2, CXCL3, CXCL8, and CXCL12 can act as autocrine growth factors in various cancers such as melanoma, adenocarcinoma, glioblastoma, leukemia, and colon, gastric, hepatic, and pancreatic cancers. ${ }^{27}$ Burger et $\mathrm{al}^{28}$ have also shown the potential implication of chemokine receptors, especially CXCR2, in the malignant transformation process. Other research groups also have demonstrated that chemokine and chemokine receptor expression could be involved in metastasis. For example, preliminary data regarding the involvement of CXCL8 in invasive melanoma or prostate cancers has been reported, ${ }^{29,30}$ as well as CXCR4 in breast cancer ${ }^{31}$ or CXCR3 in colon cancer. ${ }^{32}$ Finally, another essential role of chemokines in tumor biology is their implication in angiogenesis, required for tumor growth. CXCL1, CXCL2, CXCL3, CXCL5, CXCL8, and CXCL12 have variously been implicated in the induction of angiogenesis of several tumors. In contrast, some chemokines (e.g., CXCL4, CXCL10, and CXCL9) may inhibit this process. ${ }^{18}$

\section{CHEMOKINES AND CHEMOKINE RECEPTORS IN THE CENTRAL NERVOUS SYSTEM}

Because of their involvement in diverse neurological diseases, interest in chemokines and chemokine receptors in the CNS has been rapidly increasing. Nonetheless, the implication of the chemokine system in the physiological or pathological conditions of the CNS has only begun to be clarified.

We have already noted the extensive involvement of chemokines and chemokine receptor in CNS development. CXCL12-CXCR4 signaling controls the migration and survival of neural precursors. ${ }^{33}$ CXCL1-CXCR2 have also been implicated in the migration and proliferation of oligodendrocyte progenitors. ${ }^{34}$ A recent study, ${ }^{35}$ using a CXCR2 knockout model, reported the importance of this chemokine receptor in the maintenance of oligodendrocyte lineage, myelination, and white matter in the CNS. In parallel to this implication in CNS patterning and developmental positioning, chemokines and chemokine receptors act as physiological neuromodulators. Several studies have demonstrated that chemokines CXCL1, CXCL8, or CXCL12 regulate neurotransmitter release or modulate ion channel activity at both the presynaptic and postsynaptic levels. ${ }^{36,37}$ Moreover, a recent study has reported that CX3CL1, a chemokine constitutively expressed in the CNS (along with CXCL12 and CXCL14), is a potent neuromodulator of evoked excitatory synaptic transmission. ${ }^{38}$

Beyond their role in the CNS under physiological conditions, chemokines and chemokine receptors are studied primarily as mediators of CNS pathologies, especially those with an inflammatory component such as multiple sclerosis (MS). During neurological diseases, the expression of chemokines can be selectively induced or upregulated in a wide range of cells, including microglia, astrocytes, neurons, and endothelial cells. ${ }^{9,39}$ These 
molecules-both chemokines and chemokine receptors-represent potential therapeutic targets. In the next sections, we discuss several illustrative CNS therapeutic targets within the chemokine system.

\section{CHEMOKINES AND CHEMOKINE RECEPTORS AS BIOLOGICAL MARKERS OF NEUROLOGICAL DISEASES}

\section{CNS tumors: the case of CXCL12-CXCR4}

As we have noted, chemokines and chemokine receptors are involved in tumor biology by regulating cell proliferation, angiogenesis and metastasis. Several studies have demonstrated the important role of CXCR4CXCL12 in the biology of the most aggressive type of primary brain tumor, glioblastoma multiforme (GBM) (also known as grade 4 astrocytoma). Expression of CXCR4 has been shown in the endothelial cells of neovessels, with a high expression of its ligand in tumor cells adjacent to these neovessels, suggesting a role of CXCL12 in promoting angiogenesis. ${ }^{40} \mathrm{~A}$ correlation between the CXCR4 expression and the invasiveness of tumor cells has been reported in a wide range of cancers (e.g., breast cancer, ${ }^{41}$ melanoma, ${ }^{42}$ or prostate cancer ${ }^{43}$ ), in addition to GBMs. ${ }^{44}$ CXCL12 has also been involved in the survival of glioma cells by activating the Akt pathway. ${ }^{45}$

These findings identify CXCR4-CXCL12 as potential prognostic biomarkers for GBMs, which display heterogeneity in regard to invasiveness, angiogenesis, and extent of necrosis. Studies analyzing the cellular and genetic changes which occur during the genesis and progression of human gliomas have demonstrated the overexpression of CXCR4 in GBM tissue as compared with normal brain tissue. ${ }^{46,47}$ Rempel et al. ${ }^{40}$ demonstrated a correlation between tumor grade and the expression of CXCR4 and its ligand CXCL12. Using immunohistochemistry, they found low level expression of CXCL12 and CXCR4 in lower grade GBM tumors and higher level expression of CXCR4 and CXCL12 in higher grade GBMs, which are characterized by large regions of angiogenesis and necrosis. These data suggest that CXCL12 and CXCR4 expression could be a useful marker for grading GBMs. In addition, a more recent article $^{48}$ reported a relation between the expression of CXCL12 and a significantly shorter time to tumor progression in low-grade glioma, suggesting a potential role of CXCL12 as a marker of early disease progression.

\section{Alzheimer's disease: the case of CCR1}

Alzheimer's disease (AD), the most commonly diagnosed dementia, is characterized by neuronal loss in cortical and subcortical regions, $\beta$-amyloid $(\mathrm{A} \beta)$ peptide plaque deposits, and neurofibrillary tangles. AD pathology is associated with inflammation in the form of mi- croglial and astroglial reaction. Descriptive studies have demonstrated the presence of chemokines and their receptors in AD tissues. One study revealed elevated expression of CCR3 and CCR5 on reactive microglia, associated with amyloid deposits. ${ }^{49}$ CCR5 ligands CCL3 and CCL4 were detected also in neurons and a subpopulation of reactive astrocytes. ${ }^{49} \mathrm{CXCR} 3$ was detected on neurons, and its ligand, CXCL10, was increased in astrocytes in AD brain tissues. ${ }^{50}$ Like CXCR3, CXCR2 was expressed on neurons, with its expression strongly upregulated in a subpopulation of neuritic plaques. ${ }^{51}$ CCL2 was found in mature, senile plaques and reactive microglia of AD brain tissues. ${ }^{52}$ Moreover, in vitro studies suggest that $\mathrm{A} \beta$ peptides stimulate chemokine production by cultured microglia. ${ }^{53}$

Halks-Miller et al. ${ }^{54}$ reported a specific expression of CCR1 in dystrophic neurites and neurons in AD lesions associated with amyloid plaques - this expression being undetectable in control brain or normal-appearing brain parenchyma of AD patients. Furthermore, the expression of CCR1 was observed at a very early time point in the disease and increased with progression of severity. These results suggest that CCR1 may be an early marker of AD-associated A $\beta 1-42$ containing plaques. This study (on 86 autopsy-derived brains, including 40 cases of AD) presented novel and promising results-which need, however, to be confirmed in an additional cohort.

As already noted, A $\beta$ peptide plaque deposits characterized $\mathrm{AD}$ pathology. Positron emission tomography (PET), using radiotracers with high affinity for $\mathrm{A} \beta$ peptide plaque deposits, is a noninvasive and promising technique for AD diagnosis (from other form of dementia) and for study of disease progression and therapeutic efficiency. At present, several radiotracers have demonstrated their relevance by their retention in senile plaques of AD patients. ${ }^{55-57}$ Incorporation of radioactive tracer into small-molecule compounds that bind CCR1 with high affinity could lead to PET ligands, which would offer the novel possibility of detecting CCR1 in association with dystrophic neurites and complement the amyloid-binding compounds.

\section{Multiple sclerosis: a jumble of chemokines and chemokine receptors}

MS is an inflammatory, demyelinating disorder of the CNS. The roles of chemokines and chemokine receptors in MS pathogenesis have been widely investigated using blood cells, brain sections, cerebrospinal fluid (CSF) samples, or experimental autoimmune encephalomyelitis (EAE), an animal model of MS-associated inflammation. Analyses of the expression of chemokines and their receptors in MS have highlighted the complexity of this field, in that a large number of these molecules have been found to be involved in the trafficking of leukocytes. ${ }^{58}$ 
Several studies have investigated the expression of chemokines and chemokine receptors in the blood of MS patients. Significant increase of the CCR5 and CXCR3 expression on $\mathrm{T}$ lymphocytes in MS patients compared with controls has been reported, ${ }^{59}$ as well as a higher secretion of CXCL8 from peripheral mononuclear cells, especially monocytes. ${ }^{60}$ CCR7 and CXCR3 are expressed in the CSF by virtually all $\mathrm{T}$ lymphocytes. Apparent enrichment for CCR5 on CSF $\mathrm{T}$ cells merely reflects selective accumulation of memory cells in this compartment. ${ }^{9,61}$ In MS patients and controls, CSF monocytes express both CCR5 and CCR1, but only a small minority of blood monocytes express CCR $5{ }^{62}$ Sorensen et al. ${ }^{63}$ found elevated expression of CXCL10 and CCL5 in the CSF of MS patients, whereas CCL2 level was significantly decreased. Interestingly, this selective downregulation of CCL2 in the CSF of MS patients is not observed in noninflammatory neurological disorders, nor in other acute or chronic neuroinflammatory diseases, including stroke and HIV-1-associated encephalopathy. CSF CCL2 was also reduced (compared to nonneurological controls) in chronic neuroinflammatory disorders like HTLV-1-associated myelopathy.

An in vitro study suggested that the decrease of CCL2 in MS CSF could be a consequence of CCL2 consumption by CCR2-positive migrating cells, which then downregulate the expression of their receptors as they cross the blood-brain barrier in response to CCL2. ${ }^{64}$ Studies of CNS tissues revealed that the vast majority of perivascular lymphocytes express CXCR3. ${ }^{59,63}$ In parallel, CXCL10 (the appropriate ligand for CXCR3) is expressed by astrocytes and macrophages in MS lesions of the brain. ${ }^{59}$ Moreover, in MS lesions, mononuclear phagocytes have been described to express CCR1 and CCR5 (as already noted). The expression of chemokines CCL2, CCL3, CCL4, CCL5, CCL7, and CCL8 was also demonstrated in MS lesions. ${ }^{58,63}$

By studying two of the four patterns of demyelination in active MS lesions, ${ }^{65}$ Mahad et al. ${ }^{66}$ showed that the number of infiltrating monocytes expressing CCR 1 is decreased and the number expressing CCR5 is increased in late active demyelinating regions of pattern II lesions. Conversely, the number of cells expressing CCR1 and CCR5 are similar in all regions of pattern III lesions. Another study suggests that the expression of CX3CR1 by NK cells is associated with disease activity. ${ }^{67}$ For now, however, none of the chemokine system molecules have been characterized as a specific marker of MS physiopathology.

Additional information has been provided by EAE studies. Using monophasic or relapsing EAE models, functional roles for CXCL1, CXCL10, CCR1, and CCR2 were observed during the acute phase; CCL2, CCR2, CCL20, and CCR6 were associated with relapses. ${ }^{39}$

In addition, some studies have reported a possible correlation between susceptibility, age of onset, or severity of disease in patients who display heterozygosity for the CCR5 $\Delta 32$ mutation. ${ }^{68-70}$ The findings are contradictory, however, and more recent work using a cohort of $221 \mathrm{MS}$ patients failed to detect an association between CCR5 32 mutation and disease severity or age of onset. $^{71}$

\section{Modulating the chemokine system: consequences for cell trafficking}

Chemokines and chemokine receptors are promising potential therapeutic targets. At the same time, because of their many functions and their complex interactions (a large number of molecules with different temporal and spatial expression patterns), using the chemokine system as a therapeutic target is challenging: Which elements to target? how to do so? and when to apply these therapeutics?

Several approaches are available for modulating chemokines and chemokine receptors, of which small-molecule, peptide, and neutralizing-antibody chemokine receptor antagonists represent the most highly developed. The identification of appropriate targets for MS has followed descriptive tissue analysis and research using gene targeting or antagonist-mediated blockade in mice with EAE.

For one example, tissue studies showed a large number of CCR1-positive mononuclear phagocytic cells associated with demyelinating plaques. Using myelin oligodendrocyte glycoprotein (MOG)-induced EAE and CCR $1^{-1-}$ mice, an important role for CCR1 was demonstrated in EAE pathogenesis. ${ }^{72}$ Moreover, treatment with the CCR1 antagonist BX- $471^{73}$ produced positive effects on clinical and histological scores in a rat EAE model, supporting its therapeutic potential in MS. However, a phase I/II clinical trial of 105 relapsing-remitting MS patients who received oral CCR1 antagonist BX-471 or placebo gave negative results, in that numbers and sizes of acutely inflamed MS brain lesions (detected by gadolinium-enhanced magnetic resonance imaging $\left(\mathrm{Gd}^{+}\right.$ MRI) were equivalent in patients receiving the CCR1 antagonist or inactive placebo. ${ }^{74}$

The underlying reason for the failure of this widely anticipated trial is uncertain. Simplistically, it may be possible that CCR1 is not a suitable therapeutic target for MS treatment, in which case trial design would be irrelevant. Alternatively, it is plausible that the trial design failed to address the role of CCR1 in the pathogenesis of MS. One red flag is that EAE models used in the preclinical testing for CCR1 were all monophasic, so that disease pathogenesis more nearly resembled acute disseminated encephalomyelitis (ADEM) than MS. The distribution of $\mathrm{CCR}^{+}$cells within MS lesions (at the borders of actively demyelinating lesions) may suggest a role for this receptor in generating tissue injury in these 
lesions, rather than in leukocyte recruitment. If this hypothesis is correct, then imaging techniques that addressed lesion evolution ${ }^{75}$ might be preferable for monitoring therapeutic effects of CCR1 blockade, as opposed to quantifying $\mathrm{Gd}^{+}$MRI lesions. The distinction between these two possibilities can be made only by additional clinical trial endeavors, using either BX471 or other CCR1 antagonists.

The general take-home message is that clinical trial design for chemokine receptor blockade in MS patients must be developed in recognition that chemokine receptors are pleiotropic. Chemokine receptor functions beyond leukocyte chemoattraction may frequently play important roles in disease pathogenesis, and will require ingenious and individualized trial design strategies to capture these effects.

$\mathrm{CCR} 2^{-1-}$ mice were also shown to be resistant to MOG-induced EAE. ${ }^{76}$ An oral antagonist to CCR2 (INCB3344) $^{77}$ was evaluated in a murine EAE model and inhibited macrophage accumulation in a dose-dependent manner and reduced disease severity. No study results have been reported in MS patients.

CCR5 has been intensively studied because of its implication in HIV infection and the unique genetic studies enabled by the presence of a common null allele in humans. ${ }^{78}$ The potential role of CCR5 as a therapeutic target for MS has been reduced both by clinical and experimental observations. In particular, $\mathrm{CCR} 5^{-1-}$ mice exhibit the same susceptibility as wild-type mice to MOG-induced EAE. ${ }^{79}$ Furthermore, using an N-terminal modified human CCL5 molecule (Met-RANTES) as antagonist of CCR1 and CCR5, Matsui et al. ${ }^{80}$ reported that Met-RANTES did not alter the susceptibility to EAE, the clinical score during the acute phase and chronic-relapsing phase or leukocyte trafficking. However, Met-RANTES modestly reduced neurological disability during the chronic-plateau phase of EAE. ${ }^{81}$

As described previously, CXCR3 is present on virtually all perivascular lymphocytes in MS lesions, suggesting an important role for this chemokine receptor in directing $\mathrm{T}$ lymphocytes to sites of neuroinflammation. CXCR3 appeared to represent an exciting therapeutic target for MS disease, whose blockade might restrict the infiltration of pathogenic leukocytes into the CNS. Studies of EAE in $\mathrm{CXCR}^{-1-}$ mice, ${ }^{82}$ however, failed to show any alteration in numbers or lineage of CNS-infiltrating leukocytes. Moreover, an exaggerated disease severity associated with an increase of the blood-brain barrier disruption and a reduction of T-cell IFN $\gamma$ production were demonstrated in CNS tissues of $\mathrm{CXCR}^{-1-}$ mice with EAE.

As already noted, a critical role of CXCL12-CXCR4 has been proposed for brain tumor pathogenesis. In vitro and in vivo (xenograft mouse model) studies using the CXCR4 antagonist AMD3100 supported this concept by showing an inhibition of glioblastoma growth via increased apoptosis and decreased proliferation. ${ }^{83}$ Studies using AMD3100 have also been conducted in the EAE model. ${ }^{84}$ Animals treated with this CXCR4 antagonist displayed worsened clinical disease and extensive demyelination, although numbers of mononuclear cells were similar in CNS tissues and in vehicle-treated mice. The AMD3100-treated mice, however, showed an increase in microglial activation and remarkably dispersed intraparenchymal lymphocyte infiltrates. These observations suggest that CXCL12-CXCR4 retained mononuclear cells in the perivascular space and limited intraparenchymal inflammation. Because of this potential anti-inflammatory role, CXCR4 antagonists, which appear potentially applicable for treating glial tumors, may not represent appropriate therapeutics for CNS autoimmune disease.

The possible anti-inflammatory roles of CXCL12CXCR4 suggest that increased expression could be a potential strategy for limiting CNS inflammation. A protective and anti-inflammatory role of CX3CL1 has also been suggested in $\mathrm{AD} .{ }^{85,86}$ Cardona et al., ${ }^{87}$ using three different in vivo models, also showed that the inhibition of CX3CR1 dysregulates microglial responses resulting in neurotoxicity. These data suggest preferentially a protective role of CX3CL1-CX3CR1 signaling, and raise concerns that CNS penetration by CX3CR1 antagonists might increase neuronal vulnerability. EAE in CX3CR $1^{-\prime-}$ mice showed exaggerated disease severity, associated with an impairment of the migration of regulatory NK cells to the CNS. ${ }^{88}$ These results, and corollary studies, suggest a protective role of NK cells during EAE, and showed further that CX3CL1-CX3CR1 govern the migration of these cells to the CNS, but not to the liver.

Taken together, these data show the significant and daunting complexity of the chemokine system, posing challenges to the use of chemokine research for identifying therapeutic targets for neurological diseases. In specific diseases, some receptors exert pathogenic effects and require therapeutic blockade, whereas others are beneficial and could be upregulated. Moreover, one chemokine is often capable of binding multiple receptors, and individual receptors may be expressed on varied cell types. Finally, chemokines display pleiotropic functions. Thus, blocking one chemokine receptor to treat neurological disease could present unexpected results. Furthermore, following on evidence of an antagonist effect in an animal model, difficulties frequently occur in validating the same molecule in humans. The chemokine system is not strictly orthologous between humans and rodents, and many disease models are imperfect. 
In spite of the many difficulties, however, several molecules are undergoing testing in clinical trials. ${ }^{89}$ And, despite the magnitude of the challenge, the promise of translating chemokine biology to practice sustains our efforts to comprehend the implication of chemokines and their receptors in the pathogenesis of neurological disease.

Acknowledgments: Research in the Ransohoff laboratory has been supported by U.S. National Institutes of Health (grants R01 NS32151, P01 NS38667, and K24 NS51400), the National Multiple Sclerosis Society, the Charles A. Dana Foundation, the Robert Packard Foundation for ALS Research at Johns Hopkins University, the Boye Foundation, the Nancy Davis Center Without Walls, and the Williams Family Foundation for MS Research.

\section{REFERENCES}

1. Yoshimura T, Matsushima K, Tanaka S, et al. Purification of a human monocyte-derived neutrophil chemotactic factor that has peptide sequence similarity to other host defense cytokines. Proc Natl Acad Sci U S A 1987;84:9233-9237.

2. Holmes WE, Lee J, Kuang WJ, Rice GC, Wood WI. Structure and functional expression of a human interleukin-8 receptor. Science 1991;253:1278-1280.

3. Zlotnik A, Yoshie O. Chemokines: a new classification system and their role in immunity. Immunity 2000;12:121-127.

4. Luster AD. Chemokines: chemotactic cytokines that mediate inflammation. N Engl J Med 1998;338:436-445.

5. Laing KJ, Secombes CJ. Chemokines. Dev Comp Immunol 2004; 28:443-460.

6. Van Coillie E, Van Damme J, Opdenakker G. The MCP/eotaxin subfamily of CC chemokines. Cytokine Growth Factor Rev 1999; 10:61-86.

7. Kelner GS, Kennedy J, Bacon KB, et al. Lymphotactin: a cytokine that represents a new class of chemokine. Science 1994;266:13951399.

8. Bazan JF, Bacon KB, Hardiman G, et al. A new class of membrane-bound chemokine with a CX3C motif. Nature 1997;385: 640-644.

9. Charo IF, Ransohoff RM. The many roles of chemokines and chemokine receptors in inflammation. N Engl J Med 2006;354: $610-621$.

10. Clark-Lewis I, Kim KS, Rajarathnam K, et al. Structure-activity relationships of chemokines. J Leukoc Biol 1995;57:703-711.

11. Cartier L, Hartley O, Dubois-Dauphin M, Krause KH. Chemokine receptors in the central nervous system: role in brain inflammation and neurodegenerative diseases. Brain Res Brain Res Rev 2005; 48:16-42.

12. Mellado M, Rodríguez-Frade JM, Mañes S, Martínez-A C. Chemokine signaling and functional responses: the role of receptor dimerization and TK pathway activation. Annu Rev Immunol 2001;19:397-421.

13. Vroon A, Heijnen CJ, Kavelaars A. GRKs and arrestins: regulators of migration and inflammation. J Leukoc Biol 2006;80:12141221 .

14. Imhof BA, Engelhardt B, Vadas M. Novel mechanisms of the transendothelial migration of leukocytes. Trends Immunol 2001; 22:411-414.

15. Engelhardt B, Ransohoff RM. The ins and outs of T lymphocyte trafficking to the CNS: anatomical sites and molecular mechanisms. Trends Immunol 2005;26:485-495.

16. Middleton J, Patterson AM, Gardner L, Schmutz C, Ashton BA. Leukocyte extravasation: chemokine transport and presentation by the endothelium. Blood 2002;100:3853-3860.

17. Schreiber TH, Shinder V, Cain DW, Alon R, Sackstein R. Shear flow-dependent integration of apical and subendothelial chemo- kines in T-cell transmigration: implications for locomotion and the multistep paradigm. Blood 2007;109:1381-1386.

18. Le Y, Zhou Y, Iribarren P, Wang JM. Chemokines and chemokine receptors: their manifold role in homeostasis and disease. Cell Mol Immunol 2004;1:95-104.

19. Mackay CR. Chemokines: immunology's high impact factors. Nat Immunol 2001;2:95-101.

20. Nakano H, Mori S, Yonekawa H, Nariuchi H, Matsuzawa A, Kakiuchi T. A novel mutant gene involved in T-lymphocyte-specific homing into peripheral lymphoid organs on mouse chromosome 4. Blood 1998;91:2886-2895.

21. Forster R, Mattis AE, Kremmer E, Wolf E, Brem G, Lipp M. A putative chemokine receptor, BLR1, directs B cell migration to defined lymphoid organs and specific anatomic compartments of the spleen. Cell 1996;188:373-386.

22. Gerhardt CC, Romero IA, Cancello R, Camoin L, Strosberg AD. Chemokines control fat accumulation and leptin secretion by cultured human adipocytes. Mol Cell Endocrinol 2001;175:81-92.

23. Reddy S, Bai Y, Robinson E, Ross J. Immunolocalization of monocyte chemoattractant protein-1 in islets of NOD mice during cyclophosphamide administration. Ann N Y Acad Sci 2006;1079: 103-108.

24. Kanda N, Shimizu T, Tada Y, Watanabe S. IL-18 enhances IFNgamma-induced production of CXCL9, CXCL10 and CXCL11 in human keratinocytes. Eur J Immunol 2007;37:338-350.

25. Tachibana K, Hirota S, Lizasa H, et al. The chemokine receptor CXCR4 is essential for vascularization of the gastrointestinal tract. Nature 1998;393:591-594.

26. Zou YR, Kottman AH, Kuroda M, Taniuchi I, Littman D. Function of the chemokine receptor CXCR4 in haematopoiesis and in cerebellar development. Nature 1998;393:595-599.

27. Bendall L. Chemokines and their receptors in disease. Histol Histopathol 2005;20:907-926.

28. Burger M, Burger JA, Hoch RC, Oades Z, Takamori H, Schraufstatter IU. Point mutation causing constitutive signaling of CXCR2 leads to transforming activity similar to Kaposi's sarcoma herpes virus-G protein-coupled receptor. J Immunol 1999;163:20172022.

29. Luca M, Huang S, Gershenwald J, Singh R, Reich R, Bar-Eli M. Expression of interleukin- 8 by human melanoma cells up-regulates MMP-2 activity and increases tumor growth and metastasis. Am J Pathol 1997;151:1105-1113.

30. Inoue K, Slaton J, Eve B, et al. Interleukin-8 expression regulates tumorigenicity and metastases in androgen-independent prostate cancer. Clin Cancer Res 2000;6:2104-2119.

31. Darash-Yahana M, Pikarsky E, Abramovitch R, et al. Role of high expression levels of CXCR4 in tumor growth, vascularization and metastasis. FASEB J 2004;18:1240-1242.

32. Kawada K, Hosogi H, Sonoshita M, et al. Chemokine receptor CXCR3 promotes colon cancer metastasis to lymph nodes. Oncogene 2007;26:4679-4688.

33. Dziembowska M, Tham TN, Lau P, Vitry S, Lazarini F, DuboisDalcq M. A role of CXCR4 signaling in survival and migration of neural and oligodendrocyte precursors. Glia 2005;50:258-269.

34. Tsai HH, Frost E, To V, et al. The chemokine receptor CXCR2 controls positioning of oligodendrocyte precursors in developing spinal cord by arresting their migration. Cell 2002;110:373-383.

35. Padovani-Claudio DA, Liu L, Ransohoff RM, Miller RH. Alterations in the oligodendrocyte lineage, myelin and white matter in adult mice lacking the chemokine receptor CXCR2. Glia 2006;54: 471-483.

36. Giovannelli A, Limatola C, Ragozzino D, et al. CXC chemokines interleukin-8 (IL8) and growth-related gene product $\alpha(\mathrm{GRO} \alpha)$ modulate Purkinje neuron activity in mouse cerebellum. J Neuroimmunol 1998;92:122-132.

37. Limatola C, Giovanelli A, Maggi L, et al. SDF- $1 \alpha$-mediated modulation of synaptic transmission in rat cerebellum. Eur J Neurosci 2000;12:2497-2504.

38. Bertollini C, Ragozzino D, Gross C, Limatola C, Eusebi F. Fractalkine/CX3CL1 depresses central synaptic transmission in mouse hippocampal slices. Neuropharmacology 2006;51:816-821.

39. Ubogu EE, Cossoy MB, Ransohoff RM. The expression and func- 
tion of chemokines involved in CNS inflammation. Trends Pharmacol Sci 2006;27:48-55.

40. Rempel SA, Dudas S, Ge S, Gutierrez JA. Identification and localization of the cytokine SDF1 and its receptor, CXC chemokine receptor 4, to regions of necrosis and angiogenesis in human glioblastoma. Clin Cancer Res 2000;6:102-111.

41. Muller A, Homey B, Soto $\mathrm{H}$, et al. Involvement of chemokine receptors in breast cancer metastasis. Nature 2001;410:50-56.

42. Bartolome RA, Galvez BG, Longo N, et al. Stromal cell-derived factor- $1 \alpha$ promotes melanoma cell invasion across basement membranes involving stimulation of membrane-type 1 matrix metalloproteinase and Rho GTPase activities. Cancer Res 2004;64:25342543.

43. Singh S, Singh UP, Grizzle WE, Lillard JW Jr. CXCL12-CXCR4 interactions modulate prostate cancer cell migration, metalloproteinase expression and invasion. Lab Invest 2004;84:1666-1676.

44. Ehtesham M, Winston JA, Kabos P, Thompson RC. CXCR4 expression mediates glioma cell invasiveness. Oncogene 2006;25: 2801-2806.

45. Zhou Y, Larsen PH, Hao C, Yong VW. CXCR4 is a major chemokine receptor on glioma cells and mediates their survival. J Biol Chem 2002;277:49481-49487.

46. Sehgal A, Boynton AL, Young RF, et al. Application of the differential hybridization of Atlas human expression arrays technique in the identification of differentially expressed genes in human glioblastoma multiforme tumor tissue. J Surg Oncol 1998;67:234242.

47. Sehgal A, Ricks S, Boynton A, Warrick J, Murphy GP. Molecular characterization of CXCR4: a potential brain tumor-associated gene. J Surg Oncol 1998;69:239-248.

48. Salmaggi A, Gelati M, Pollo B, et al. CXCL12 expression is predictive of a shorter time to tumor progression in low-grade glioma: a single-institution study in 50 patients. J Neurooncol 2005;74:287-293.

49. Xia MQ, Qin SX, Wu LJ, Mackay CR, Hyman BT. Immunohistochemical study of the beta-chemokine receptors CCR3 and CCR5 and their ligands in normal and Alzheimer's disease brains. Am J Pathol 1998;153:31-37.

50. Xia MQ, Bacskai BJ, Knowles RB, Qin SX, Hyman BT. Expression of the chemokine receptor CXCR3 on neurons and the elevated expression of its ligand IP-10 in reactive astrocytes: in vitro ERK1/2 activation and role in Alzheimer's disease. J Neuroimmunol 2000;108:227-235.

51. Xia M, Hyman BT. GRO $\alpha / \mathrm{KC}$, a chemokine receptor CXCR2 ligand, can be a potent trigger for neuronal ERK1/2 and PI-3 kinase pathways and for tau hyperphosphorylation: a role in Alzheimer's disease? J Neuroimmunol 2002;122:55-64.

52. Ishizuka K, Kimura T, Igata-yi R, Katsuragi S, Takamatsu J, Miyakawa T. Identification of monocytes chemoattractant protein-1 in senile plaques and reactive microglia of Alzheimer's disease. Psychiatry Clin Neurosci 1997;51:135-138.

53. Streit WJ, Conde JR, Harrison JK. Chemokines and Alzheimer's disease. Neurobiol Aging 2001;22:909-913.

54. Halks-Miller M, Schroeder ML, Haroutunian V, et al. CCR1 is an early and specific marker of Alzheimer's disease. Ann Neurol 2003;54:638-646.

55. Shoghi-Jadid K, Small GW, Agdeppa ED, et al. Localization of neurofibrillary tangles and beta-amyloid plaques in the brains of living patients with Alzheimer disease. Am J Geriatr Psychiatry 2002;10:24-35.

56. Klunk WE, Engler H, Nordberg A, et al. Imaging brain amyloid in Alzheimer's disease with Pittsburgh compound-B. Ann Neurol 2004;55:306-319.

57. Verhoeff NP, Wilson AA, Takeshita S, et al. In-vivo imaging of Alzheimer disease beta-amyloid with $\left[{ }^{11} \mathrm{C}\right] \mathrm{SB}-13$ PET. Am J Geriatr Psychiatry 2004;12:584-595.

58. Trebst C, Ransohoff RM. Investigating chemokines and chemokine receptors in patients with multiple sclerosis. 2001;58:19751980.

59. Balashov KE, Rottman JB, Weiner HL, Hancock VW. CCR $5^{+}$and $\mathrm{CXCR}^{+}{ }^{+} \mathrm{T}$ cells are increased in multiple sclerosis and their ligands MIP- $1 \alpha$ and IP-10 are expressed in demyelinating brain lesions. Proc Natl Acad Sci U S A 1999;96:6873-6878.
60. Lund BT, Ashikian N, Ta HQ, et al. Increased CXCL8 (IL-8) expression in multiple sclerosis. J Neuroimmunol 2004;155:161171.

61. Teleshova N, Pashenkov M, Huang YM, et al. Multiple sclerosis and optic neuritis: CCR5 and CXCR3 expressing T cells are augmented in blood and cerebrospinal fluid. J Neurol 2002;249:723729 .

62. Trebst C, Sorensen TL, Kivisakk P, et al. $\mathrm{CCR} 1^{+} / \mathrm{CCR} 5^{+}$ mononuclear phagocytes accumulate in the central nervous system of patients with multiple sclerosis. Am J Pathol 2001;159: 1701-1710.

63. Sorensen TL, Tani M, Jensen J, et al. Expression of specific chemokines and chemokine receptors in the central nervous system of multiple sclerosis patients. J Clin Invest 1999;103:807-815.

64. Mahad D, Callahan MK, Williams KA, et al. Modulating CCR2 and CCL2 at the blood-brain barrier: relevance for multiple sclerosis pathogenesis. Brain 2006;129:212-223.

65. Lucchinetti C, Bruck W, Parisi J, Scheithauer B, Rodriguez M, Lassman H. Heterogeneity of multiple sclerosis lesions: implications for the pathogenesis of demyelination. Ann Neurol 2000;47: 707-717.

66. Mahad DJ, Trebst C, Kivisakk P, et al. Expression of chemokine receptors CCR1 and CCR5 reflect differential activation of mononuclear phagocytes in pattern II and pattern III multiple sclerosis lesions. J Neuropathol Exp Neurol 2004;63:262-273.

67. Infante-Duarte C, Weber A, Kratzschmar J, et al. Frequency of blood CX3CR1-positive natural killer cells correlates with disease activity in multiple sclerosis patients. FASEB J 2005;19:19021904.

68. Sellebjerg F, Madsen HO, Jensen CV, Jensen J, Garred P. CCR5 $\Delta 32$, matrix metalloproteinase- 9 and disease activity in multiple sclerosis. J Neuroimmunol 2000;102:98-106.

69. Gade-Andavolu R, Comings DE, MacMurray J, et al. Association of CCR5 $\Delta 32$ deletion with early death in multiple sclerosis. Genet Med 2004;6:126-131.

70. Kantor R, Bakhanashvili M, Achiron A. A mutated CCR5 gene may have favorable prognostic implications in MS. Neurology 2003;61:238-240.

71. Kantarci OH, Morales Y, Ziemer PA, et al. CCR5 532 polymorphism effects on CCR5 expression, patterns of immunopathology and disease course in multiple sclerosis. J Neuroimmunol 2005; 169:137-143

72. Rottman JB, Slavin AJ, Weiner HL, Gerard CG, Hancock WW. Leukocyte recruitment during onset of experimental allergic encephalomyelitis is CCR1 dependent. Eur J Immunol 2000;30:2372-2377.

73. Liang M, Mallari C, Rosser M, et al. Identification and characterization of a potent, selective, and orally active antagonist of the CC chemokine receptor-1. J Biol Chem 2000;275:19000-19008.

74. Zipp F, Hartung HP, Hillert J, et al. Blockade of chemokine signaling in patients with multiple sclerosis. Neurology 2006;67: $1880-1883$.

75. Ge Y. Seeing is believing: in vivo evolution of multiple sclerosis pathology with magnetic resonance. Top Magn Reson Imaging 2006;17:295-306.

76. Izikson L, Klein RS, Charo IF, Weiner HL, Luster A. Resistance to experimental autoimmune encephalomyelitis in mice lacking the CC chemokine receptor (CCR)2. J Exp Med 2000;192:1075-1080.

77. Brodmerkel CM, Huber R, Covington M, et al. Discovery and pharmacological characterization of a novel rodent-active CCR2 antagonist, INCB3344. J Immunol 2005;175:5370-5378.

78. Barber CG. CCR5 antagonists for the treatment of HIV. Curr Opin Investig Drugs 2004;5:851-861.

79. Tran EH, Kuziel WA, Owens T. Induction of experimental autoimmune encephalomyelitis in C57B1/6 mice deficient in either the chemokine macrophage inflammatory protein- $1 \alpha$ or its CCR5 receptor. Eur J Immunol 2000;30:1410-1415.

80. Matsui M, Weaver J, Proudfoot A, et al. Treatment of experimental autoimmune encephalomyelitis with the chemokine receptor antagonist Met-RANTES. J Neuroimmunol 2002;128:16-33.

81. Bjartmar C, Kidd G, Mork S, Rudick R, Trapp BD. Neurological disability correlates with spinal cord axonal loss and reduced $\mathrm{N}$ - 
acetyl aspartate in chronic multiple sclerosis patients. Ann Neurol 2000;48:893-901.

82. Liu L, Huang D, Matsui M, et al. Severe disease, unaltered leukocyte migration, and reduced IFN- $\gamma$ production in $\mathrm{CXCR}^{-1-}$ mice with experimental autoimmune encephalomyelitis. J Immunol 2006;176:4399-4409.

83. Rubin JB, Kung AL, Klein RS, et al. A small-molecule antagonist of CXCR4 inhibits intracranial growth of primary brain tumors. Proc Natl Acad Sci U S A 2003;100:13513-13518.

84. McCandless EE, Wang Q, Woerner BM, Harper JM, Klein RS. CXCL12 limits inflammation by localizing mononuclear infiltrates to the perivascular space during experimental autoimmune encephalomyelitis. J Immunol 2006;177:8053-8064.

85. Combs CK, Karlo JC, Kao SC, Landreth GE. Beta-amyloid stimulation of microglia and monocytes in $\mathrm{TNF} \alpha$-dependent expres- sion of inducible nitric oxide synthase and neuronal apoptosis. J Neurosci 2001;21:1179-1188.

86. Zujovic V, Benavides J, Vigé X, Carter C, Taupin V. Fractalkine modulates TNF- $\alpha$ secretion and neurotoxicity induced by microglial activation. Glia 2000;29:305-315.

87. Cardona AE, Pioro EP, Sasse ME, et al. Control of microglial neurotoxicity by the fractalkine receptor. Nat Neurosci 2006;9: 917-924.

88. Huang D, Shi FD, Jung S, et al. The neuronal chemokine CX3CL1/ fractalkine selectively recruits NK cells that modify experimental autoimmune encephalomyelitis within the central nervous system. FASEB J 2006;20:896-905.

89. Wells TNC, Power CA, Shaw JP, Proudfoot AEI. Chemokine blockers: therapeutics in the making? Trends Pharmacol Sci 2006; 27:41-47. 